\title{
Gastric Dieulafoy's lesion in an elderly man with comorbidities: A case report
}

\author{
Ibrahim Alnashri ${ }^{1}$, Abdullah Aljohani1 ${ }^{1}$, and Mohamed Eldemouhi²
}

1. Department of Internal Medicine, King Fahad Hospital, Jeddah, Saudi Arabia

2. Department of Gastroenterology, King Fahad Hospital, Jeddah, Saudi Arabia

\section{CASE STUDY}

Please cite this paper as: Alnashri I, Aljohani A, Eldemouhi M. Gastric Dieulafoy's lesion in an elderly man with comorbidities: A case report. AMJ 2020;13(2):46-49. https://doi.org/10.35841/1836-1935.13.2.46-49

\section{Corresponding Author:}

Ibrahim Alnashri

Department of Internal Medicine, King Fahad Hospital, Jeddah, Saudi Arabia

Email: ialnashri@hotmail.com

\section{ABSTRACT}

Dieulafoy's lesion (DL) is an abnormal, enlarged (approximately $1-3 \mathrm{~mm}$ ) and tortious submucosal artery in the gastrointestinal tract wall. Herein, we report a case of recurrent bleeding from a gastric fundus $D L$ in a 59-year-old man who presented with a history of melena associated with dizziness and palpitations. Esophagogastroduodenoscopy revealed a tortuous, dilated vessel in the gastric fundus. Computed tomography angiography revealed an aberrant vessel branching from the celiac artery. Surgery was performed, resulting in complete resolution of the patient's symptoms. DL is a rare but lifethreating cause of gastrointestinal haemorrhage and should be considered in the differential diagnosis.

\section{Key Words}

Dieulafoy's lesion, gastrointestinal bleeding, esophagogastroduodenoscopy

\section{Implications for Practice:}

\section{What is known about this subject?}

$\mathrm{DL}$ is an underrecognised cause of gastrointestinal bleeding, representing less than 5 per cent of all causes of gastrointestinal bleeding.

\section{What new information is offered in this case study?}

DL poses a high risk of recurrent bleeding regardless of the mucosal integrity. Thus, early therapeutic interventions should be considered.

3. What are the implications for research, policy, or practice?

All patients presenting with obscured gastrointestinal bleeding should be carefully investigated for $\mathrm{DL}$, particularly in case of present comorbidities or risk factors for DL.

\section{Background}

Dieulafoy's lesion (DL) is an abnormal, enlarged (approximately $1-3 \mathrm{~mm}$ ) and tortious submucosal artery in the gastrointestinal tract (GIT) wall. The French surgeon Paul Dieulafoy first described this disease in his scientific paper published in 1896. It is also known as Dieulafoy's ulcer, gastric arteriosclerosis, gastric aneurysm, submucosal arterial malformation, cirsoid aneurysm, calibre persistent artery, and solitary exulceratio simplex. ${ }^{1} \mathrm{DL}$ accounts for up to 5 per cent of all acute GIT bleeding causes. ${ }^{1}$ It is usually found in the first part of the gastric wall, approximately $5 \mathrm{~cm}$ from the gastro-oesophageal junction, but it can be found in all parts of the GIT, including the oesophagus, small and large intestines. ${ }^{2,3}$ It occurs more commonly in males, older adults, and individuals with comorbidities, such as diabetes mellitus, hypertension, cardiovascular disease, and chronic kidney disease. ${ }^{3-6}$ Recently, alcohol consumption and antiplatelet agents were reported as risk factors for DL formation. ${ }^{5}$ The clinical manifestation of $\mathrm{DL}$ includes melena, haematemesis, haematochezia, tachycardia, hypotension, and syncope. ${ }^{7}$ Bleeding episodes are often self-limited, although the bleeding can be recurrent, profuse, or life-threatening. Herein, we report a case of gastric fundus $\mathrm{DL}$ in a 59-year-old man.

\section{Case details}

A 59-year-old Saudi male patient with a history of diabetes mellitus and hypertension presented to the hospital with a 1-week history of melena, dizziness, and palpitations. He had been taking aspirin daily for the last one year as a 
primary prophylaxis. On admission, his pulse rate was $118 \mathrm{bpm}$, blood pressure was $128 / 78 \mathrm{~mm} \mathrm{Hg}$, respiratory rate was $18 \mathrm{breaths} / \mathrm{min}$, oxygen saturation was 94 per cent on room air, and body temperature was $36.8^{\circ} \mathrm{C}$. Physical examination revealed pale conjunctivas, mild epigastric tenderness, and hepatomegaly. Laboratory findings on admission were as follows: haemoglobin: $8.3 \mathrm{~g} / \mathrm{dl}$, mean corpuscular volume $88.5 \mathrm{fl}$, mean corpuscular haemoglobin $26.7 \mathrm{pg}$, white blood cell count 11.4, platelet count 336, prothrombin time $12.6 \mathrm{~s}$, partial thromboplastin time $24.2 \mathrm{~s}$, international normalized ratio 1.1 , creatinine $1.21 \mathrm{mg} / \mathrm{dl}$, blood urea nitrogen $21 \mathrm{mg} / \mathrm{dl}$, sodium $136 \mathrm{mmol} / \mathrm{L}$, potassium $4.68 \mathrm{mmol} / \mathrm{L}$, aspartate transaminase $21 \mathrm{U} / \mathrm{L}$, alanine aminotransferase $43 \mathrm{U} / \mathrm{L}$, total bilirubin $0.48 \mathrm{mg} / \mathrm{dl}$, direct bilirubin $0.12 \mathrm{mg} / \mathrm{dl}$. Tests for human immunodeficiency virus antibody, hepatitis B surface antigen, and hepatitis $\mathrm{C}$ antibody were negative. Abdominal computed tomography (CT) angiography showed a welldefined tortuous vascular structure in the gastric fundus (Figure 1) and enlarged fatty liver, measuring up to $20 \mathrm{~cm}$. Esophagogastroduodenoscopy revealed a tortuous, dilated vessel in the gastric fundus without any obvious erosions (Figure 2).

The patient's condition improved after proton pump inhibitor infusion; thus, considering the above findings, a therapeutic endoscopic or surgical intervention was not performed at that time. The patient was discharged in a good clinical condition. However, he was readmitted 1 week later due to recurrent bleeding manifesting as hematemesis. Diagnostic upper GIT endoscopy showed the presence of the same large tortoise artery, without any obvious erosions. CT angiography showed an aberrant, large, tortuous artery inside the gastric wall, which branched from the celiac artery. Therapeutic endoscopic intervention was not performed due to the large artery size and the high risk of complication occurrence. Arteriographic embolization was attempted as a safe therapeutic option; however, we could not reach the aberrant gastric artery. Therefore, we performed surgical ligation through exploratory laparotomy without any complications. At the follow-up at 1 month after surgery, the patient's symptoms had fully resolved and his haemoglobin level had improved.

\section{Discussion}

Although the aetiology of DL is still uncertain, it is considered that these lesions are congenital in nature. ${ }^{8}$ Currently, endoscopy is the main diagnostic and therapeutic method for DL. Endoscopic criteria for DL include: (1) Active arterial bleeding from a visible tiny mucosal defect or from the normal mucosa; (2) Presence of a large vessel within the
GIT wall, measuring approximately $1-3 \mathrm{~mm}$, with or without bleeding or mucosal defect; or (3) Presence of an adherent clot attached to the tiny mucosal defect or normal mucosa. ${ }^{7}$ There is no specific radiological modality used for diagnosing the disease, but barium study, video capsule endoscopy, angiography, and tagged red blood cell scanning can be used to locate the bleeding site. Endoscopic intervention is the first-line therapy for DL. ${ }^{9}$ It includes regional injection therapy, such as injection of epinephrine or sclerosants, mechanical haemostasis therapy, such as band ligation or application of haemoclips, and thermal coagulation therapy, such as heat probe coagulation or electrocoagulation. ${ }^{10}$ The rate of bleeding recurrence is lower in mechanical haemostasis therapy than in injection therapy, ${ }^{11}$ but using them together gives better results in controlling the bleeding, preventing recurrent bleeding, and decreasing hospital stay lenght. ${ }^{12}$ Surgical ligation can be considered for failed endoscopic intervention. Angiography with embolization is another alternative modality that can be considered in patients with active bleeding who cannot tolerate endoscopic and surgical interventions. ${ }^{13}$ After therapeutic interventions, the patient should be followed up for at least six months due to the high risk of recurrent bleeding during this period. ${ }^{14}$ In the present case, recurrent bleeding occurred one week after the initial conservative treatment. At that time, endoscopic treatment was deemed unsafe due to the size of the aberrant artery, and arteriographic embolization failed. Therefore, surgical intervention was performed with a good clinical outcome.

\section{Conclusion}

Although DL is uncommon, it should be considered in the differential diagnosis of GIT bleeding, particularly in case of present comorbidities or risk factors, because it poses a high risk of recurrent bleeding regardless of the mucosal integrity. The risk of undiscovered DL in another part of the GIT should also be considered, particularly in patients with ulcers. Early intervention is the mainstay in DL management. Combining two different endoscopic intervention methods provides best treatment outcomes. Arteriography with embolization can be considered in some cases. Surgical intervention is preferred when the patient is hemodynamically unstable or when other methods have failed.

\section{References}

1. Chaer RA, Helton WS. Dieulafoy's disease. J Am Coll Surg. 2003;196(2):290-6. https://doi.org/10.1016/S10727515(02)01801-X 
2. al-Mishlab T, Amin AM, Ellul JP. Dieulafoy's lesion: an obscure cause of $\mathrm{Gl}$ bleeding. J R Coll Surg Edinb. 1999;44(4):222-5. PMID: 10453143.

3. Lara LF, Sreenarasimhaiah J, Tang SJ, et al. Dieulafoy lesions of the GI tract: localization and therapeutic outcomes. Dig Dis Sci. 2010;55(12):3436-41. https://doi.org/10.1007/s10620-010-1385-0.

4. Arora A, Mehrotra R, Patnaik PK, et al. Dieulafoy's lesion: a rare cause of massive upper gastrointestinal haemorrhage. Trop Gastroenterol. 1991;12(1):25-30. PMID: 2058007.

5. Shin HJ, Ju JS, Kim KD, et al. Risk factors for Dieulafoy lesions in the upper gastrointestinal tract. Clin Endosc. 2015;48(3):228-33. https://doi.org/10.5946/ce.2015.48.3.228.

6. Linhares MM, Filho BH, Schraibman V, et al. Dieulafoy lesion: endoscopic and surgical management. Surg Laparosc Endosc Percutan Tech. 2006;16(1):1-3. https://doi.org/10.1097/01.sle.0000202191.59322.5f.

7. Stark ME, Gostout CJ, Balm RK. Clinical features and endoscopic management of Dieulafoy's disease. Gastrointest Endosc. 1992;38(5):545-50. https://doi.org/10.1016/s0016-5107(92)70513-6.

8. Schmulewitz N, Baillie J. Dieulafoy lesions: A review of 6 years of experience at a tertiary referral center. Am J Gastroenterol. 2001;96(6):1688-94. https://doi.org/10.1111/j.1572-0241.2001.03922.x.

9. Fujishiro $M$, Iguchi $M$, Kakushima $N$, et al. Guidelines for endoscopic management of non-variceal upper gastrointestinal bleeding. Dig Endosc. 2016;28(4):36378. https://doi.org/10.1111/den.12639.

10. Jaspersen D. Dieulafoy's disease controlled by Doppler ultrasound endoscopic treatment. Gut. 1993;34:857-8. https://doi.org/10.1136/gut.34.6.857.

11. Chung IK, Kim EJ, Lee MS, et al. Bleeding Dieulafoy's lesions and the choice of endoscopic method: comparing the hemostatic efficacy of mechanical and injection methods. Gastrointest Endosc. 2000;52(6):721-4. https://doi.org/10.1067/mge.2000.108040.

12. Chereau N, Chandeze MM, Tantardini C, et al. Antroduodenectomy with gastroduodenal anastomosis: salvage emergency surgery for complicated peptic ulcer disease--Results of a double institution study of 35 patients. J Gastrointest Surg. 2016;20(3):539-45. https://doi.org/10.1007/s11605-015-3050-6.

13. Katz PO, Salas L. Less frequent causes of upper gastrointestinal bleeding. Gastroenterol Clin North Am. 1993;22(4):875-89. PMID: 8307643.

14. Baettig B, Haecki W, Lammer F, et al. Dieulafoy's disease: endoscopic treatment and follow up. Gut
1993;34(10):1418-21.

https://doi.org/10.1136/gut.34.10.1418.

\section{PEER REVIEW}

Not commissioned. Externally peer reviewed.

\section{CONFLICTS OF INTEREST}

The authors declare that they have no competing interests.

\section{FUNDING}

None

\section{PATIENT CONSENT}

The authors, Alnashri I, Aljehani A, Eldemouhi $M$, declare that:

1. They have obtained written, informed consent for the publication of the details relating to the patient(s) in this report.

2. All possible steps have been taken to safeguard the identity of the patient(s).

3. This submission is compliant with the requirements of local research ethics committees. 
Figure 1: Transverse view of abdominal CT angiogram

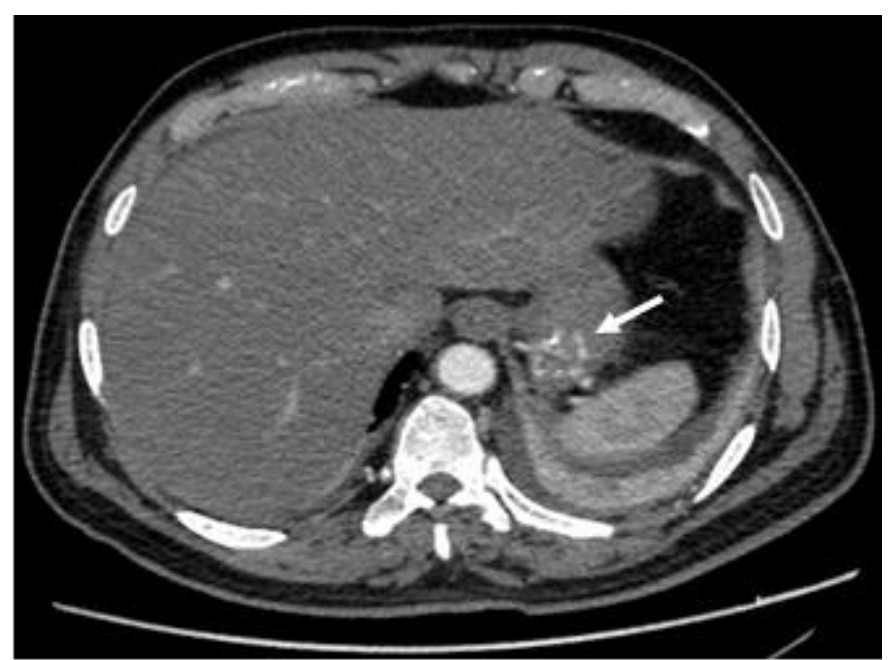

Note: A prominent vessel within the gastric wall (arrow)

Figure 2: Endoscopic view of the gastric fundus Dieulafoy's lesion
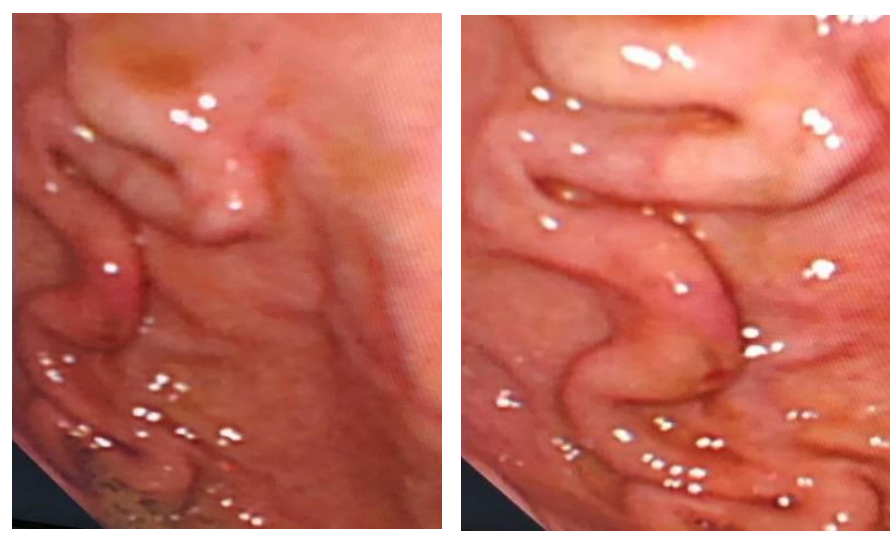

Note: A large, tortuous vessel prominent through the gastric fundus mucosa without any obvious defects or bleeding 